\title{
Developing a Comprehensive Patient Safety Strategy for an Integrated Canadian Healthcare Region
}

\author{
$\overline{\text { W. Ward Flemons, Chris J. Eagle and Jack C. Davis }}$
}

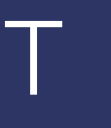

he Calgary Health Region (the Region) is one of the largest completely integrated health regions in Canada, covering an area of 39,260 square kilometres. With four urban hospitals and eight rural hospitals (total of 2,104 acute care beds), the Region provides population health, preventive health, acute care, long-term care and home care services for 1.14 million residents, as well as tertiary care services for residents of southern Alberta, southwestern British Columbia and south-eastern Saskatchewan.

In 2004, a batch of citrated renal dialysis solution was mistakenly prepared by the Region's central pharmacy with potassium chloride rather than sodium chloride. Two patients in our critical care units undergoing continuous renal replacement therapy who were dialyzed with this solution developed severe hyperkalemia and subsequently died. The Region immediately recalled the remaining dialysate solutions and also informed other acute care sites in Canada that produced this type of dialysate about the adverse event. A subsequent internal review led to changes in the storage and labelling of potassium chloride in the central pharmacy and also to the dialysate solution production process. The Region disclosed the details of the adverse events to the families of the involved patients and shortly thereafter, with their permission, the Region informed the public. The Region commissioned a comprehensive external review of its pharmacy services and its organizational approach to patient safety. From this, and through its own internal processes, the Region has developed a comprehensive organizational patient safety strategy.

In the last several years, many excellent reports have recommended approaches for improving healthcare safety (Institute of Medicine 2001; National Steering Committee on Patient Safety 2002; Institute of Medicine 2004). However, we were unable to find a report or guide written specifically for large integrated health regions that provided a comprehensive "how to" roadmap to address the complex area of patient safety. The internal and external reviews suggested opportunities that would assist the Region in creating a safer environment for patients. An important outcome of these efforts was the production of a framework that highlighted the key areas that needed to be addressed (Figure 1), and which has also served as the basis for the creation of the Region's patient safety strategy. We acknowledged that in its broadest context patient safety encompasses occupational safety, environmental safety, physical plant and equipment safety, and business risk management, as well as clinical safety (i.e., the day-to-day practices that directly impact patients). Our patient safety strategy, however, deals only with the narrower context of "clinical safety." 
Figure 1. The Calgary Health Region's Safety Framework

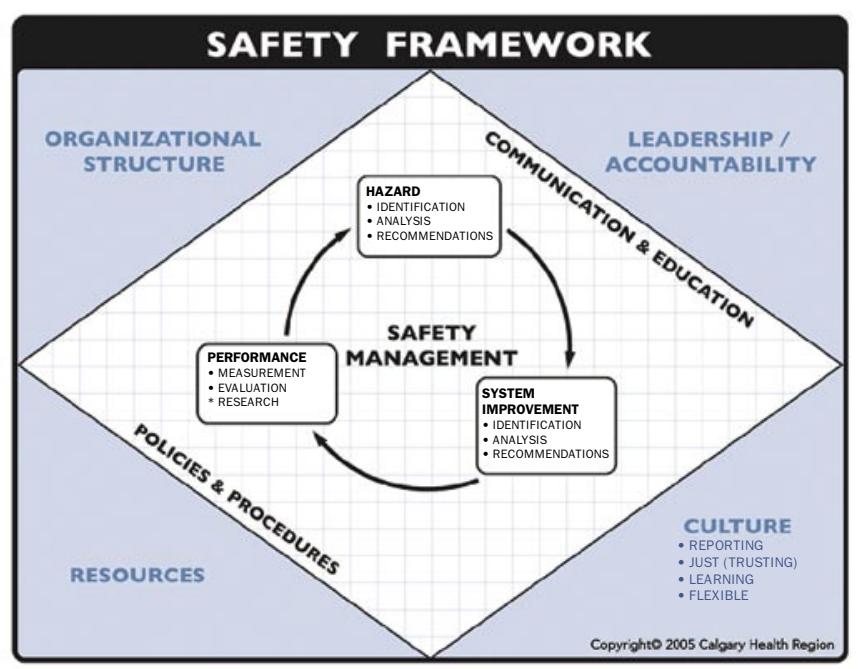

\section{Safety Management}

The core piece of our Region's strategy is a cycle of safety management that starts with developing and formalizing hazard identification processes. These processes include the reporting of adverse events and close calls, investigations of critical adverse events and close calls, leadership walkrounds (Frankel et al. 2003), adverse event audits, mortality audits, focus groups, as well as safety alerts received from other organizations (e.g., the Institute for Safe Medication Practice). Once hazards are identified they require analysis and management including: (1) better understanding of the contributing factors, (2) prioritization, and (3) recommendations for system improvements that mitigate risks for patients. Analyses can be informal or structured, for example, when an adverse event or close call is reviewed using a root cause analysis framework (Bagian et al. 2002) or where a detailed process review is undertaken using a failure modes and effects analysis (DeRosier et al. 2002). The Calgary Health Region has chosen not to use a standard root cause analysis framework for reviews of adverse events or close calls. Instead, the Region is using a human factors approach adapted from aviation safety by one of our academic anaesthesia colleagues (Davies and Lange 2003). We refer to this approach as a health system safety analysis.

Mitigating risks to patients through system improvements encompasses both structural changes and process changes. Examples of structural changes include alterations in staffing, equipment and workspace. Process changes include all of the critical elements that are part of clinical process design or redesign that ensures reliable delivery of evidence-based care, for example, correct timing of antibiotic prophylaxis to prevent surgical site infections or rapid reperfusion of patients suffering an acute myocardial infarction.
The final part of our safety management cycle involves continuously checking the performance of the system through:

1. a set of safety performance outcome or process measures;

2. a formal evaluation of the system improvements that have been recommended (i.e., whether they have been implemented as planned, whether they have had the desired effect and whether they have resulted in creating unanticipated risk), and

3. researching new methods of delivering safer healthcare.

While we view safety management as the core piece of an overarching strategy in our Region, we believe there are four cornerstones that provide the foundation for long-term success: (1) committed and engaged leadership, (2) a supportive organizational structure, (3) a culture of safety, and (4) access to appropriate resources.

\section{Leadership/Accountability}

Regional/Hospital boards and their management teams have a key role in ensuring appropriate management for safety in their organizations. The Calgary Health Region's Board established a Safety Task Force to oversee the Region's safety strategy. The Region recently reconfigured its balanced scorecard to highlight quality and safety and is reviewing its mission and vision with the goal of better capturing patient safety. Leadership walkrounds (Frankel et al. 2003) have been initiated. Patient safety events are actively promoted and supported by the Executive Management Team, members of which routinely participate in forums and conferences. Assigning accountability for addressing safety issues and implementing recommendations has become more formalized; reports tracking progress on implementing safety recommendations are produced for management and are shared with the board.

\section{Organizational Structure}

The Region has created an integrated structure to exclusively address safety issues in response to a key observation of the external safety review. The Region's five clinical portfolios and all key organizational support areas are represented on a newly established Regional Clinical Safety Committee (Figure 2). In addition, each clinical portfolio is establishing a clinical safety committee that will address portfolio-specific issues and represent the clinical departments and service areas within that portfolio on the regional committee. Department-based quality assurance committees, with traditional physician-only membership, are being transformed into multidisciplinary service clinical safety committees that report to their respective portfolio. Pilot testing of unit-based safety action teams (Morath and Turnbull 2005) that are linked to appropriate service clinical safety committees has also started. 
Figure 2. The Calgary Health Region's Structure and Membership of Its Regional Clinical Safety Committee

\section{REGIONAL CLINICAL SAFETY COMMITTEE}

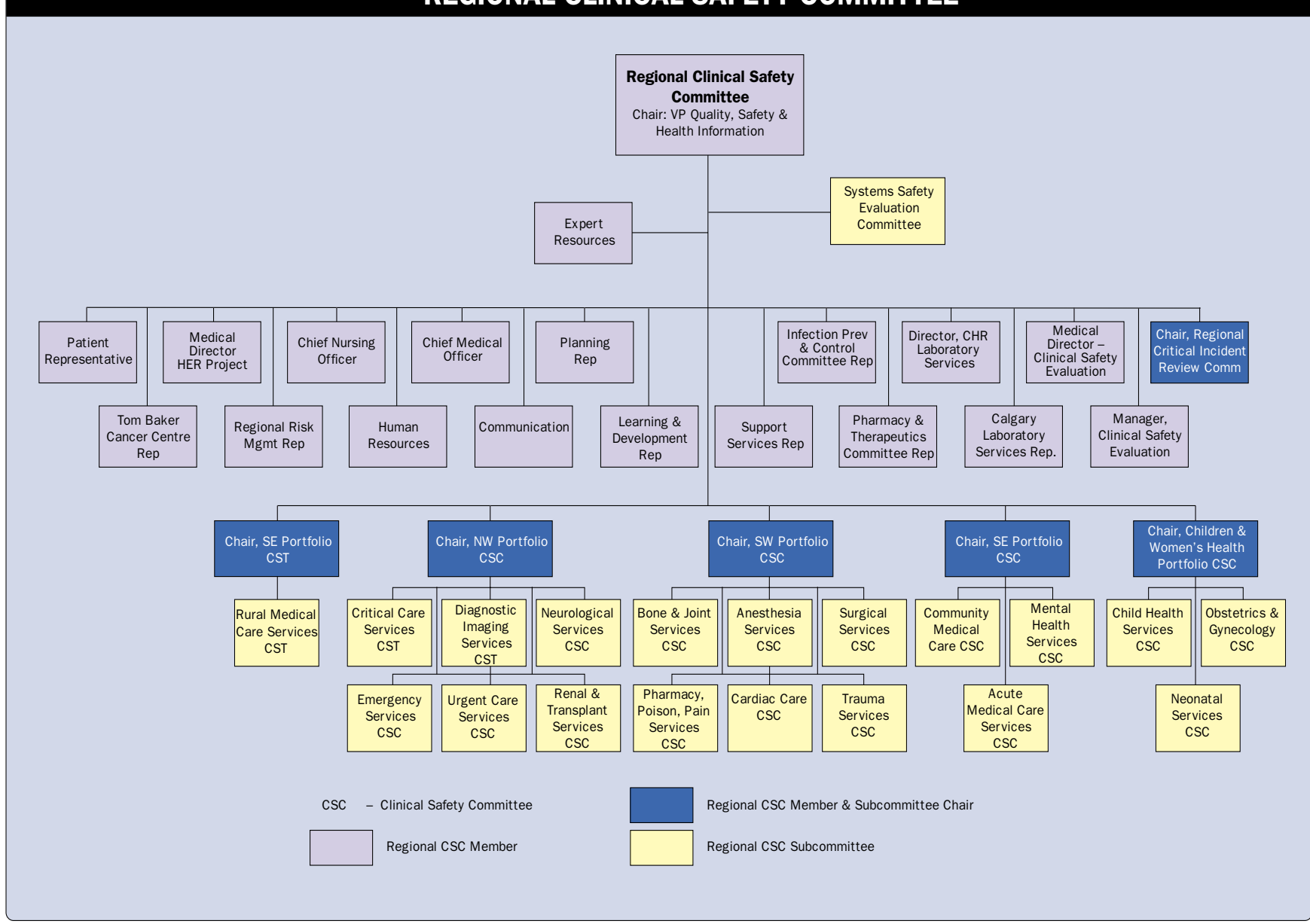

\section{Safety Culture}

To address deficiencies in our safety culture, we have adopted the approach of Reason (1997) and focused on reporting, learning, a just (and trusting) culture, and flexibility. Making improvements that address system weaknesses identified by our healthcare providers is a key goal. To create a culture where people feel safe to report hazards, we have established an organizational just and trusting culture policy and a reporting policy (see below). The reporting policy clearly outlines what the Region feels is appropriate for healthcare providers to report. The Region currently has an incident reporting process that is used mostly by employed staff and rarely by physicians. Reports predominately focus on individual behaviours (usually errors), are filed with a person's administrative supervisor and then recorded in a centralized database, which is not optimized to detect recurring system weaknesses. In our transformed reporting system, our healthcare providers will be encouraged (not required, which implies consequences for not reporting) to file "safety learning reports" (Morath and Turnbull 2005) with a focus on safety hazards, rather than incident reports. Safety learning reports will be filed, not with an immediate supervisor, but with a central reporting office that will maintain reporter confidentiality. De-identified safety reports will be available to appropriate managers so that local safety issues can be addressed in a timely manner. Feedback will be delivered to our healthcare providers about the reports they file and system improvements that result; this is a fundamental requirement to create a reporting and learning culture. The need for flexibility in our system has been addressed by providing contingency funds that can be easily accessed for making quick system improvements and by promoting a balance of local system fixes by safety action teams with region-wide system improvements for issues that affect multiple service areas. 


\section{Resources}

The Region has invested several million dollars to build the infrastructure, training, communication and equipment required to support this strategy and to provide contingency funds for ongoing system improvements. These funds allow portfolios and service areas an opportunity to quickly invest in safer systems rather than wait for the annual budgeting approval process.

In addition to the four cornerstones of our patient safety strategy, we believe that there are two additional activities that are critical for creating a safer organization: (1) having appropriate safety policies and procedures, and (2) facilitating ongoing communication and education.

\section{Policies AND Procedures}

Based on the recommendations of the external safety reviews, the Region formalized its approach to the management of potassium chloride in the form of a regional policy. In addition, to address the ethical issues of maintaining communication between the Region and its patients, its providers and its key partners and stakeholders, several policies have been developed to promote a safety culture and a culture of transparency. To avoid confusion over terminology, we defined three types of communication (Figure 3):

1. Reporting - communication between healthcare providers and the Region

2. Disclosure - communication between the Region (including its healthcare providers) and patients about circumstances when patients have been harmed by the care that they have received

3. Informing - communication between the Region and its key partners and stakeholders

Figure 3. The Relationship of Four Safety Policies on Disclosing, Reporting, Informing and Creating a Just \& Trusting Culture

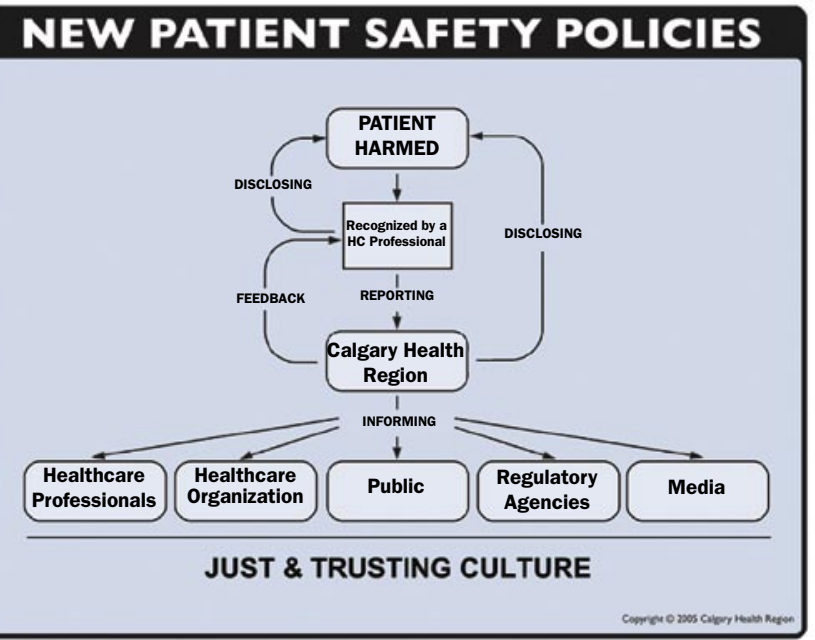

Finally, the Region developed a Just and Trusting Culture Policy to define the relationship with its healthcare providers in circumstances when care has not been appropriate and/or when patients are harmed.

\section{Reporting Policy}

The Region has defined two types of reporting that it will promote: (1) voluntary safety learning reports of hazards (including hazards that are recognized as having the potential to cause or contribute to harm but have not yet done so, situations in which patients are nearly harmed - close calls - and situations where patients are harmed but not severely), and (2) mandatory reporting when patients have suffered severe harm (defined as loss of limb or organ function or where a life sustaining intervention has been required) or fatal harm. We believe the voluntary system has the greatest potential as a source of information about where the system needs improvement to mitigate risk to patients as well as being an important vehicle to continue building the organization's safety culture.

\section{Disclosure Policy}

The Region's policy defines patient harm as an unexpected or normally avoidable outcome that negatively affects a patient's health and/or quality of life, and occurs or has occurred during the course of receiving healthcare or services from the Region (modified from the College of Physicians and Surgeons of Ontario 2003) The policy states that harm will be acknowledged, circumstances about the event will be communicated to the patient and an apology for the harm will be made. This has been a challenging policy to develop because of four important issues: (1) whether or not to mandate disclosure of close calls (the decision was made to leave this to the discretion of the primary healthcare provider(s)), (2) the concern over potential liability (for both the Region and physicians), (3) the conundrum that facts discovered during a quality assurance committee review are protected under provincial law (the Alberta Evidence Act) and cannot be revealed, and (4) the question of how to handle disclosure of harm to a patient when they suffered harm in another jurisdiction preceding the transfer of the patient to the Region. Guidelines are under development that will outline the Region's approach to these challenging issues.

The policy states that harm will be acknowledged, circumstances about the event will be communicated to the patient and an apology for the harm will be made. 


\section{Informing Policy}

This policy describes the circumstances where the Region would communicate safety issues with: (1) its principal health partners, defined as the Region's patients (inclusive of all individuals who receive healthcare or services directly from the Region), its health care providers and other healthcare providers who are not Region employees or who do not have privileges with the Region but who provide health services to the Region's patients, and (2) stakeholders (individuals and organizations that have an interest or a stake in healthcare or services including the public, and local, provincial, national and/or international healthcare providers, and health-related agencies or organizations including regulatory, non-regulatory, government bodies). This policy addresses the Region's obligations to communicate safety information where a patient's health or welfare may be at risk and where this risk may impact the health or welfare of other patients, of healthcare providers or other stakeholders. The policy recognizes how a serious adverse event has the potential to weaken the trust of the Region's principal health partners, and the most responsible approach to deal with this is to maintain an atmosphere of transparency.

\section{Just and Trusting Culture Policy}

This policy describes the Region's response to its healthcare providers who are involved in an adverse event. Our policy acknowledges two distinct types of safety evaluations:

1. Safety analyses conducted using our health system safety analysis framework with its focus on understanding systemrelated contributing factors

2. Administrative reviews conducted in situations where an evaluation of an individual healthcare provider's performance is required

We have adapted the approach to evaluating unsafe acts outlined by Reason (1997) - errors, violations and sabotage. Based on feedback from consultations undertaken with our healthcare providers, we modified Reason's terminology and refer to "non-compliance" rather than violation and "intention to harm" rather than sabotage. The Region's response to the three types of active failures will be:

- Errors - in situations where patients have been harmed in the course of receiving health care or services from the Region, or in situations where patients have been nearly harmed and where healthcare providers did not deviate from established policies, procedures, standards or guidelines, healthcare providers will not be disciplined by the Region.

- Non-compliance - in situations where patients have been harmed in the course of receiving healthcare or services from the Region, or in situations where patients have been nearly harmed and where healthcare providers have deviated from

\begin{abstract}
We have found moving forward with a comprehensive patient safety strategy very challenging because of the enormity and complexity of the task.
\end{abstract}

established policies, procedures, standards, or guidelines, the Region will commit to evaluate as part of an administrative review: (1) the appropriateness of its policies, procedures, standards or guidelines, and (2) the circumstances that led to the non-compliant action(s), before determining an appropriate course of action.

- Intention to harm - in situations where patients have been intentionally harmed or where there is intent to cause harm to a patient by any of the Region's healthcare providers, the Region will seek disciplinary action and criminal investigations may result.

\section{Communication and Education}

Breakdowns in communication are a major contributing factor in the majority of sentinel events reported to the Joint Commission of Accreditation of Healthcare Organizations (2005). The Region has begun testing a structured communication tool - SBAR - situation/background/assessment/recommendation (Leonard et al. 2004) in some of our critical care units, as well as timeouts in some of our operating theatres. A region-wide rollout of these strategies is planned. Communication is also necessary to keep the Region's healthcare providers informed about the components of the Region's patient safety strategy. We recognize that education of our healthcare providers and administrative leaders is essential to advancing the safety agenda. For several years, the Region has involved hundreds of people in collaborative projects that teach rapid cycle testing of improvements. Formal courses on quality improvement and safety theory will be offered in late 2005 including instruction in health system safety analysis and human factors analysis. Education plans will also include training on specific issues (e.g., changes in the way high hazard medications are ordered).

We have found moving forward with a comprehensive patient safety strategy very challenging because of the enormity and complexity of the task. We have found a safety framework on which to base the strategy invaluable, but are still struggling with an optimal method of prioritizing the work that needs to be accomplished and maintaining a proper perspective on the timeframe that these changes will require. Having complete organizational commitment to such a strategy is of paramount importance. 


\section{References}

Bagian, J.P., J. Gosbee, C.Z. Lee, L. Williams, S.D. McKnight and D.M. Mannos. 2002. "The Veterans Affairs Root Cause Analysis System in Action." Jt Comm J Qual Improv 28(10): 531-45.

Davies, J.M. and I.R. Lange. 2003. "Investigating Adverse Outcomes in Obstetrics." 2003. Journal of Obstetrics and Gynaecology of Canada 25: 505-15.

DeRosier, J., E. Stalhandske, J.P. Bagian and T. Nudell. 2002. "Using Health Care Failure Mode and Effect Analysis: The VA National Center for Patient Safety's Prospective Risk Analysis System." Jt Comm J Qual Improv 28(5): 248-67.

Frankel, A., E. Graydon-Baker, C. Neppl, T. Simmonds, M. Gustafson and T.K. Gandhi. 2003. "Patient Safety Leadership WalkRounds." Jt Comm J Qual Improv 29(1): 16-26.

Institute of Medicine. 2001. Crossing the Quality Chasm: A New Health System for the 21st Century. Washington D.C. National Academies Press.

Institute of Medicine. 2004. Patient Safety: Achieving a New Standard of Care. Washington D.C. National Academies Press.

Joint Commission of Accreditation of Healthcare Organizations. 2005. Root Causes of Sentinel Events. Oakbrook Terrace. Retrieved August 5, 2005. <http://www.jcaho.com/accredited+organizations/ ambulatory+care/sentinel+events/root+causes+of+sentinel+event. htm>.

Langley, G.J., K.M. Nolan, T.W. Nolan, C.L. Norman and L.P. Provost. 1996. The Improvement Guide: A Practical Approach to Enhancing Organizational Performance. San-Francisco, CA: Jossy-Bass Inc.
Leonard, M., A. Frankel, T. Simmonds and K. Vega. 2004. Achieving Safe and Reliable Healthcare: Strategies and Solutions. Chicago, Il: Health Administration Press.

Morath, J.M. and J.E.Turnbull. 2005. To Do No Harm: Ensuring Patient Safety in Health Care Organizations. San-Francisco, CA: JossyBass Inc.

National Steering Committee on Patient Safety. 2002. Building a Safer System. Royal College of Physicians and Surgeons of Canada. Ottawa. Retrieved August 5, 2005. <http://rcpsc.medical.org/publications/ building_a_safer_system_e.pdf>.

Reason, J. 1997. Managing the Risks of Organizational Accidents. Burlington, VT: Ashgate Publishing Company.

\section{About the Authors \\ W. Ward Flemons, MD, is Vice-President, Quality, Safety \& Health Information, Calgary Health Region, Calgary, Alberta, Canada.}

Chris J. Eagle, MD, is Executive Vice-President and Chief Clinical Officer, Calgary Health Region, Calgary, Alberta, Canada.

Jack C. Davis is President and Chief Executive Officer, Calgary Health Region, Calgary, Alberta, Canada.

Corresponding Author: Dr. W. Ward Flemons, Calgary Health Region, 10101 Southport Road SW, Calgary, Alberta, Canada, T2W 3N2, Voice: (403) 943-0915, Fax: (403) 943-0916 (fax), Ward.Flemons@calgaryhealthregion.ca

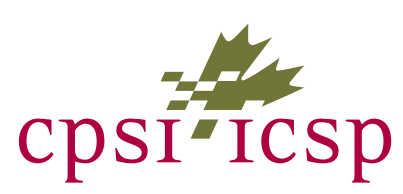 \\ Canadian Institut \\ Patient canadien \\ Safety pour la sécurité \\ Institute des patients \\ Canadian Patient Safety Week
Making Healthcare Safer Oct. 21-28, 2005}

Semaine nationale de la sécurité des patients Établir des soins de santé plus sécuritaires Du 21 au 28 octobre 2005
The Canadian Patient Safety Institute is pleased to collaborate with the Ontario Hospital Association and Longwoods Publishing to produce this special edition of Healthcare Quarterly.

We are also collaborating with our national partners to raise awareness of initiatives to improve patient safety across the country through CANADIAN PATIENT SAFETY WEEK, October 21 - 28, 2005. We hope you will participate in the first week of this annual event by sharing news of your own patient safety initiatives with your patients and their families, other organizations and the public.
L'Institut canadien pour la sécurité des patients est heureux de collaborer avec la Ontario Hospital Association et Longwoods Publishing dans la production de cette édition trimestrielle spéciale de la revue Healthcare Quarterly.

Nous collaborons également avec nos partenaires nationaux durant la SEMAINE NATIONALE DE LA SÉCURITÉ DES PATIENTS du 21 au 28 octobre 2005 afin de les sensibiliser aux diverses initiatives visant l'amélioration de la sécurité des patients. Nous espérons que vous participerez à cette semaine annuelle inaugurale, en partageant vos propres initiatives pour assurer la sécurité des patients avec vos patients, leurs familles, le grand public et d'autres organisations. 